\title{
Excessive Gingival Display - an Aesthetic Challenge; Surgical Treatment of Altered Passive Eruption
}

\section{Vrushali Abhyankar $^{1 *}$, Grant King ${ }^{2}$, Najib Ghadri ${ }^{1}$, Jules Mascarenhas ${ }^{3}$ and Paul Bland $^{4}$}

${ }^{1}$ Assistant Professor, University of Tennessee Health Science Center, College of Dentistry, 875, Union Avenue, Memphis, Tennessee, US ${ }^{2}$ Resident, Department of Periodontics, University of Tennessee Health Science Center, College of Dentistry, Union Avenue, Memphis, Tennessee, US

${ }^{3}$ Director of Graduate Periodontics, Department of Periodontics, University of Tennessee Health Science Center, College of Dentistry, Union Avenue, Memphis, Tennessee, US

${ }^{4}$ Associate Professor, Dept. of Periodontics, University of Tennessee Health Science Center, College of Dentistry, Union Avenue, Memphis, Tennessee, US

*Corresponding Author: Vrushali Abhyankar, Assistant Professor, University of Tennessee Health Science Center, College of Dentistry, Union Avenue, Memphis, Tennessee, US.

Received: July 15, 2019; Published: August 30, 2019

DOI: $10.31080 /$ ASDS.2019.03.0632

\begin{abstract}
Excessive gingival display, a condition with more than esthetically acceptable gingival visibility, negatively affects the smile and in turn the self-esteem of an individual. This article presents a case series detailing the treatment of three patients who presented with a chief complaint of a "gummy smile". After clinical examination and extra oral analysis the patients were diagnosed with altered passive eruption, altered passive eruption \& vertical maxillary excess, and altered passive eruption and hypermobile lip. Despite their different etiology or combination of etiologies, each one was treated with gingivectomy, full thickness flap, and hard tissue esthetic crown lengthening. After 6 months of healing, each patient was given the option for additional procedures including orthognathic surgery and lip repositioning to enhance smile esthetics. However, all patients were pleased with the results of the crown lengthening and declined further treatment. This approach demonstrates that favorable esthetic results and patient happiness can be achieved without the need for additional and more invasive procedures.
\end{abstract}

Keywords: Treatment; Passive Eruption; Excessive Gingival Display

\section{Introduction}

An attractive smile not only improves the overall facial attractiveness but also a person's self-esteem. A variety of factors such as deep bite, increased over jet, crowding, excessive anterior teeth display, excessive gingival display; to name a few, have a significant negative psychological impact. It is not unusual for individuals who are dissatisfied with their smile to completely avoid smiling, thereby altering the quality of life.

Excessive gingival display (EGD) commonly referred, as "gummy smile" is one such descriptive condition that may prompt treatment to improve the esthetic appearance and in effect the self-confidence $[1,2]$.
Gingival dimensions in terms of location, shape and contour affect the esthetics of the maxillary anterior region. Whether there is excessive display or gingival recession variation from normal appears unaesthetic [3]. Some amount of gingival display, up to $1 \mathrm{~mm}$ is almost necessary for a youthful appearance while 2-3mm could be aesthetically acceptable as found out in a survey of lay people [4]. 3mm of gingival display was the threshold of acceptability for Canadian and American population whereas $4 \mathrm{~mm}$ was considered acceptable for some European populations. In a study by Ker, $2 \mathrm{~mm}$ of gingival coverage over the maxillary incisors was considered ideal [5]. Gingival contour was considered ideal when the gingival margins of canines and upper central incisors were at the same level where as the lateral incisors was $1 \mathrm{~mm}$ 
coronal to that of central incisor and canine. The gingival zenith was $1 \mathrm{~mm}$ to the distal of the vertical midline, the lateral incisor was $0.4 \mathrm{~mm}$ distal while for the canine the zenith coincided with vertical midline [6]. "Gummy smile" is thus described as display of gingiva or oral mucosa beyond what is considered acceptable or esthetic when smiling and speaking, a condition recognized by the American Academy of Periodontology as a deformity and mucogingival condition that affects the area around the teeth [7].

\section{Treatment considerations}

In a sample of 450 adults, aged 20-30years, Tjan and Miller found $7 \%$ of men and $14 \%$ of women to have excessive gingival display [8]. The percentage of women having twice the display as compared to men was consistent over a number of other studies [9]. A variety of etiological causes have been proposed for EGD and a correct diagnosis of the same is essential to establish a correct treatment protocol. For instance, plaque or drug induced gingival enlargement and altered passive eruption are usually manifested clinically as an EGD. Both conditions can be treated surgically by different approaches and techniques to achieve a favorable outcome. Altered passive eruption commonly involves the need to reduce the hard tissue around teeth while plaque or drug induced gingival enlargement mainly requires soft tissue excision. In addition, EGD can result from various etiologies such as dentoalveolar extrusion, vertical maxillary excess, short or hyperactive lip which are treated with one or a combination of different methods including orthodontics, oral and periodontal surgical treatment modalities. Hence it becomes very important to come to a correct diagnosis, given the vastly varied nature of treatment options for the one condition of gummy smile [10].

A thorough examination, both extra oral and intraoral along with radiographs and diagnostic casts, is necessary to establish the etiology for EGD.

- Medical history: The patients' medical history will help to rule out drug induced gingival enlargement commonly caused due to calcium channel blockers like nifedepine, anti-seizure medications like phenytoin and immunosuppressant like cyclosporine [11].

- Facial height: Measurements of face height, lower facial height, and lip length are important diagnostic parameters of an extra oral examination. Face height is analyzed by dividing the face into thirds. The midface is measured from glabella, the most prominent point of the forehead between the eyebrows, to subnasale, the point directly below the nose. The lower face is measured from subnasale to soft tissue menton, which is the lower border of the chin.
- $\quad$ Lip length: When measured in repose, the length of the middle third of the face should equal the length of the lower third. Lip length, which is measured from sub nasale to lower border of upper lip, is usually $20-22 \mathrm{~mm}$ in young women and $22-24 \mathrm{~mm}$ in young men. In repose, $2-4 \mathrm{~mm}$ of maxillary central incisors are usually visible while in full smile [12].

- Maxillary incisor display: 3-4mm of incisor teeth display at rest and $1-2 \mathrm{~mm}$ of gingival display is common during smile or speech [9]. Special attention should be made towards the patients smile line, which is the position of the upper lip during activity like smiling, laughing or speaking. A high smile line displays the entire crown of the incisors along with a significant amount of gingiva, an average smile displays roughly 75 to 100 percent of the crowns and a low smile line may not display the incisor crowns.

- Gingival design or display: A harmonious gingival outline with the gingival zenith of central incisors and canines, $1 \mathrm{~mm}$ apical than lateral incisors is ideal. The gingival apices are located in the center of the crown or slightly distal, but changes in the apices are not known to affect the smile negatively. As discussed before, gingival display of more than $3-4 \mathrm{~mm}$ is considered unaesthetic.

- Radiographs: Apart from full mouth peri-apical series and bitewings, lateral cephalograms may be needed to rule out dentoalveolar extrusion and vertical maxillary excess. Overgrowth of maxilla in vertical direction resulting in excessive lower face height or over eruption of the anterior teeth can be noted on lateral cephalograms. A lower than normal occlusal plane is the cause of excessive gingival display and orthodontic or oral surgical corrections are the ideal treatment modalities. Gerber and Salama introduced a classification along with treatment modalities for VME in 1996 [1].

\begin{tabular}{|l|c|c|}
\hline & Gingival display & Treatment \\
\hline I & $2-4 \mathrm{~mm}$ & $\begin{array}{c}\text { Orthodontics and intrusion } \\
\text { Orthodontics and Periodontics } \\
\text { Periodontics and restorative }\end{array}$ \\
\hline II & $4-8 \mathrm{~mm}$ & $\begin{array}{c}\text { Periodontics and restorative } \\
\text { Orthognathic surgery }\end{array}$ \\
\hline III & $>8$ & $\begin{array}{c}\text { Orthognathic surgery with } \\
\text { or without periodontal and } \\
\text { restorative procedures }\end{array}$ \\
\hline
\end{tabular}

Table 1: Classification and treatment options for excessive gingival display. 
Intraoral examination: The anterior and posterior arches should be in harmony with the occlusal plane being parallel to an imaginary line connecting the lip commissures and two thirds the retro molar pad, to rule out dentoalveolar extrusion.

Incisor teeth dimensions: Assess the dimensions of anterior teeth by comparing anatomic versus clinical crown length which will determine whether short crowns are a result of incisal wear or a coronal position of the gingival margin. Clinical crown is the portion of the tooth visible clinically, while anatomic crown is the crown length from CEJ to incisal edge. Crown length of the maxillary central incisor generally measures $10-11 \mathrm{~mm}$ and can act as a good starting point for the evaluation.

Periodontal examination: This includes probing depths, distances measured from CEJ to gingival margins, attachment levels, bone level to $\mathrm{CEJ}$ measurements on radiographs, width of the keratinized tissue and biotype.

\section{Altered passive eruption}

Altered passive eruption: A term coined by Goldman in 1980 is defined as "the situation in which the gingival margin in the adult is located incisal to the cervical convexity of the crown and removed from the cemento-enamel junction of the tooth." As the free gingival margin does not recede to the expected level of CEJ, the condition possesses an esthetic challenge as well as a periodontal health risk during anterior restorations [13].

Active eruption is the actual movement of the tooth towards the occlusal plane and stops when tooth makes contact teeth the opposing arch as opposed to passive eruption which is the apical shift of dentogingival complex, which only means more of the tooth getting exposed due to the movement of gingiva. Classically, passive eruption has been classified in four stages depending on the position of the gingival complex on the tooth [14].

- Stage 1: The dentogingival junction is located on the enamel

- Stage 2: The dentogingival junction is located on the enamel as well as cementum

- Stage 3: The dentogingival junction is located on the cementum but the margin reaches the CEJ

- Stage 4: The dentogingival junction and the margin is on cementum thereby causing gingival recession, a condition is which is pathological.

Biologic width which is the dimension of soft tissue including epithelial and connective tissue attachment coronal to the alveolar crest is about $2.04 \mathrm{~mm} \mathrm{[15].} \mathrm{It} \mathrm{has} \mathrm{been} \mathrm{shown} \mathrm{that} 3 \mathrm{~mm}$, constituting $1 \mathrm{~mm}$ supracrestal connective tissue attachment, $1 \mathrm{~mm}$ junctional epithelium and $1 \mathrm{~mm}$ for gingival sulcus on an average, between the preparation margin and alveolar bone maintains periodontal health for 4 to 6 months [16]. This $3 \mathrm{~mm}$ constitutes also for adequate biologic width even when the restoration margins are placed $0.5 \mathrm{~mm}$ within the gingival sulcus or $3 \mathrm{~mm}$ from CEJ when no restorations are to be planned.

\section{Clinical reports}

Case 1:

A 23 year old healthy female patient reported to department of periodontics, University of Tennessee Health Science Center with the chief complaint of a gummy smile. She was very dissatisfied with amount of gingiva showing and had a restrictive smile. Her treatment goal was to reduce the gingival display and smile without consciously trying to cover her gums with the upper lip. She displayed $6-8 \mathrm{~mm}$ of gingiva on an exaggerated smile with visibility of teeth extending from right first molar to left first molar. The lip length was normal and not hyperactive. Intraoral evaluation revealed discrete short clinical crowns in the maxillary anterior segment. Intraoral periapical radiographs and trans gingival probing revealed alveolar crest within $1 \mathrm{~mm}$ of CEJ. The length of the maxillary anterior clinical crowns were as follows- Left and right canines-8mm, left and right lateral incisiors-7 $\mathrm{mm}$ and left and right central incisors were $9 \mathrm{~mm}$. Short clinical crowns, alveolar crest within $1 \mathrm{~mm}$ of CEJ, excessive gingival display and normal lip length confirm a final diagnosis of altered passive eruption.

Surgical treatment: Treatment option considered for altered passive eruption was surgical exposure of maxillary anterior crowns under local anesthesia. (Lignocaine $\mathrm{HCl}$ with $2 \%$ epinephrine 1:100,000) The height of the alveolar crest was marked through trans gingival probing by marking 3 bleeding on each tooth. Internal bevel gingivectomy was carried out with to expose the complete anatomic crowns up to the CEJ, taking care that the gingival contour was scalloped appropriately and followed the CEJ. It is important to establish the biologic width otherwise the proximity of the alveolar crest to the gingival margin will result in tissue rebound to naturally reestablish the biologic width.

Full thickness flap was elevated buccally from maxillary left $1^{\text {st }}$ molar to maxillary right $1^{\text {st }}$ molar extending beyond the mucogingival junction. Osseous crest at the CEJ or within $1 \mathrm{~mm}$ was observed. Osseous resective procedure including osteoplasty and ostectomy was completed to establish a $3 \mathrm{~mm}$ of biologic width i.e. osseous resection was carried out in such way that the new crest followed the scalloped contour and was $3 \mathrm{~mm}$ from the CEJ. High speed round diamonds and hand instruments like chisels and files 
were used for osseous resection. Flaps were re approximated after copious irrigation, with single interrupted vicryl sutures. Patient was prescribed analgesic and anti-inflammatory medicines and chlorhexidine mouthwash but no antibiotics and discontinue brushing. Sutures were removed at one week post op and patient was asked to continue brushing with soft tooth brush.

Reestablishment of stable dentogingival complex is usually seen 6 months after treatment, which is a suitable timeframe to begin restorative treatment if required.

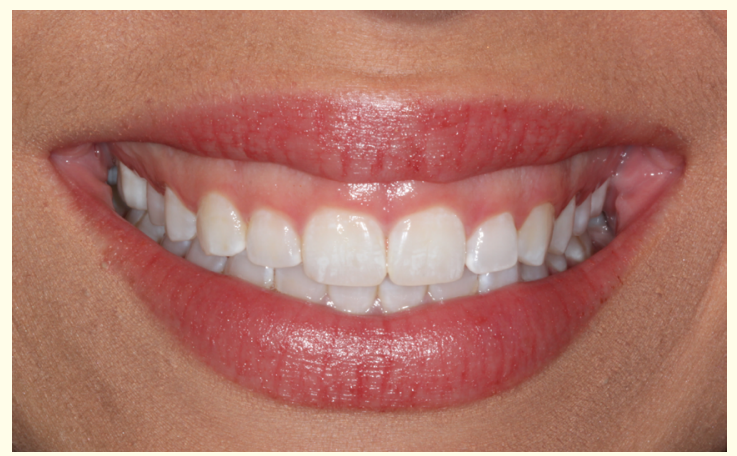

Figure 1: Pre-op smile showing clinical crowns 2-3 mm shorter than ideal (altered passive eruption).

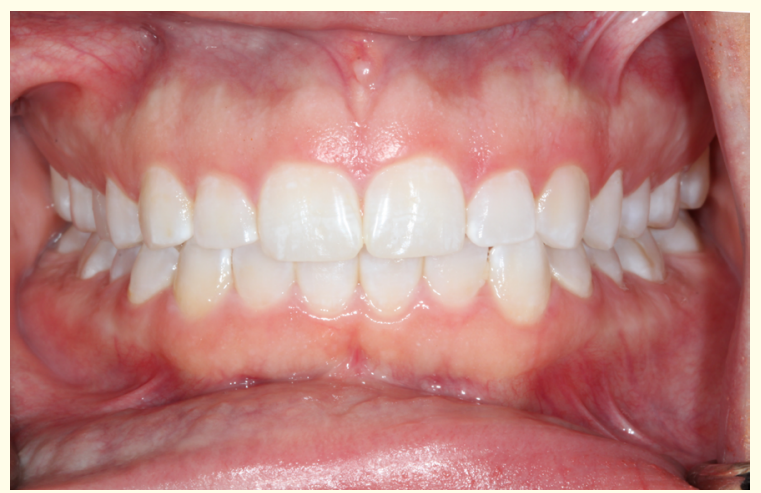

Figure 2: Pre-op retracted showing short clinical crowns.

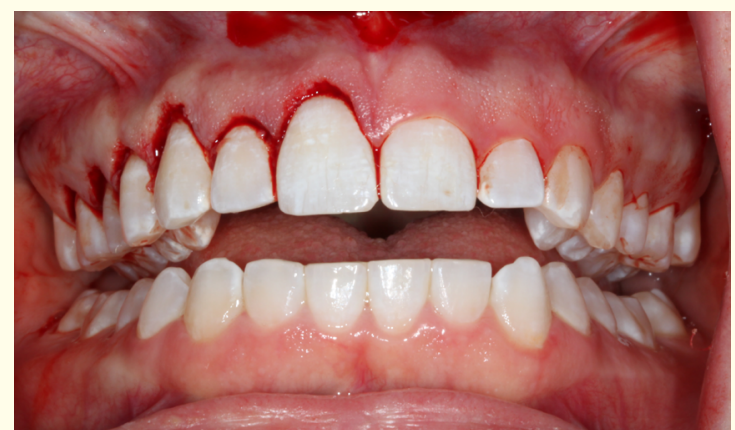

Figure 3: Gingivectomy to establish approximate location of new gingival margins and ideal tooth display.

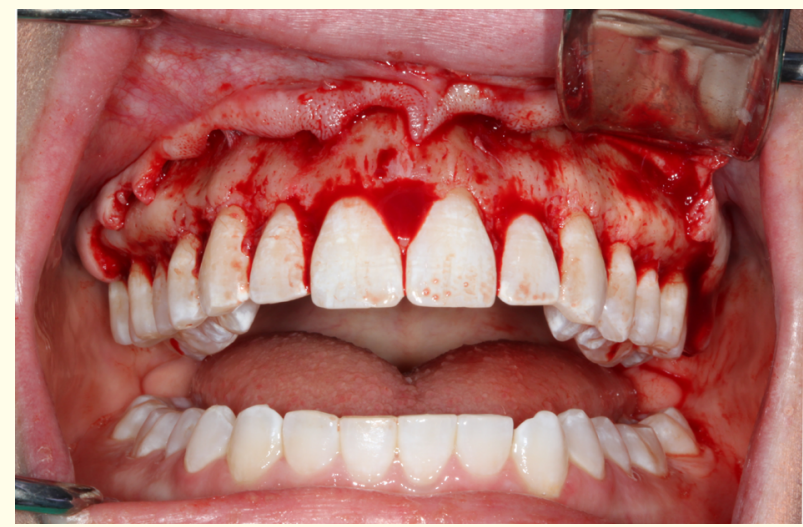

Figure 4: Pre-osseous recontouring with labial alveolar bone approximating CEJ and thick buccal shelf posteriorly.

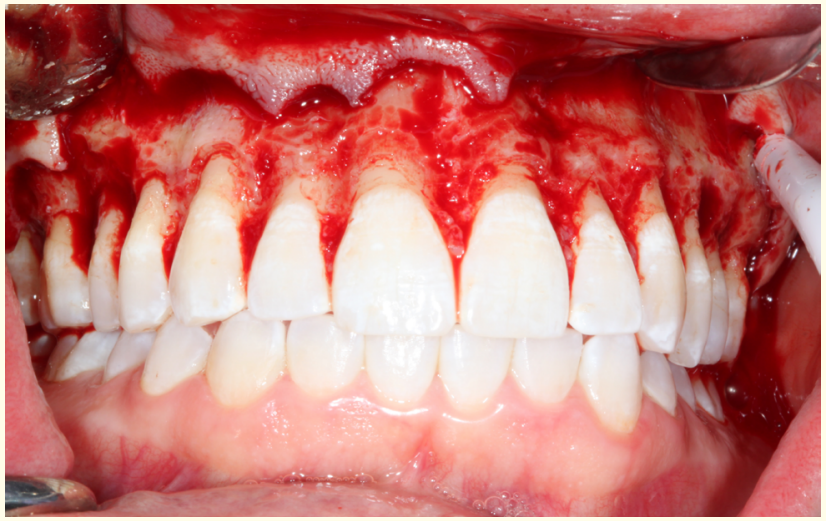

Figure 5: Post-ostectomy and osteoplasty eliminated thick bony shelves posteriorly and positioned alveolar crest $3 \mathrm{~mm}$ from CEJ.

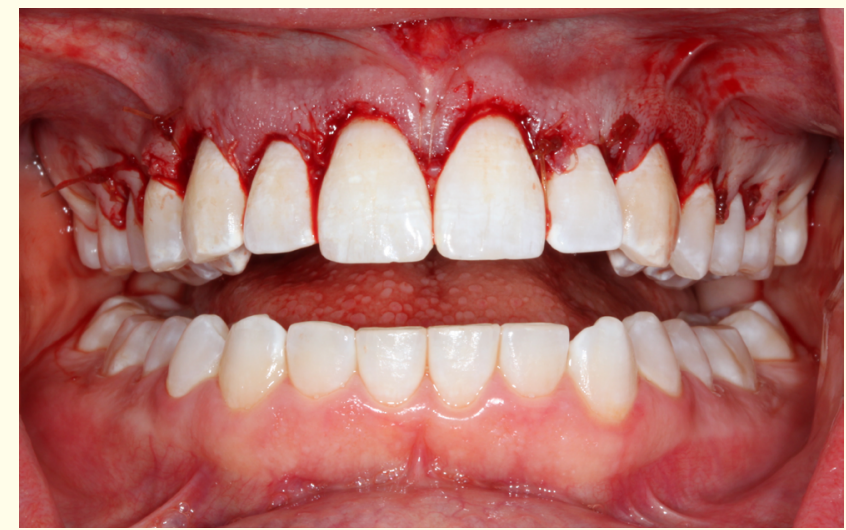

Figure 6: Suturing (single interrupted sling sutures to position margins and interproximal papilla). 


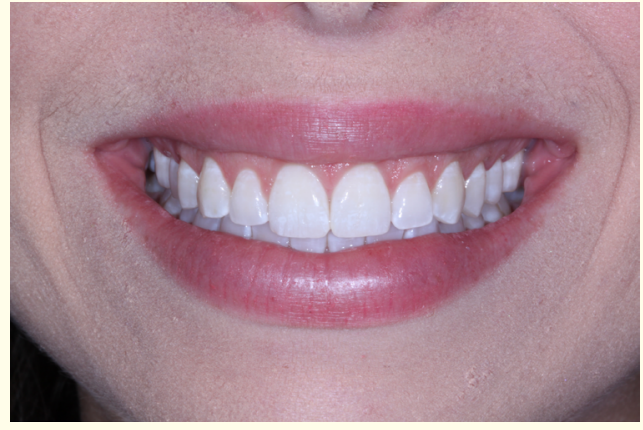

Figure 7: 6 month post-op smile.

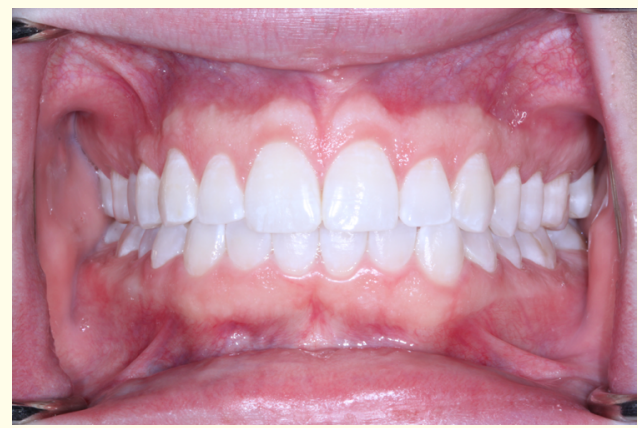

Figure 8: 6 month post-op retracted.

Case 2:

31 year old female with chief complaint of "gummy smile". Patient presented with excessive gingival display during high smile or laughing, less than ideal tooth lengths, slight incisal wear, and uneven gingival margins and marginal contours. CEJ was not detected within gingival sulcus. After clinical examination and facial analysis patient diagnosed with altered passive eruption and hypermobile lip. Patient was offered esthetic crown lengthening, followed by lip repositioning in the event the patient was not satisfied with esthetic crown lengthening result. After healing, patient is very pleased with results of crown lengthening procedure and was not interested in additional procedures.

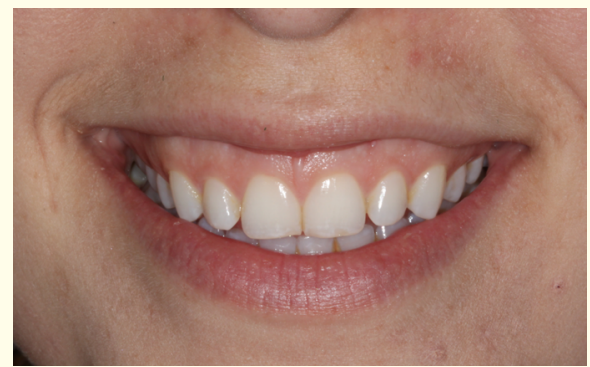

Figure 9: Pre-op smile clinical crowns $\sim 2 \mathrm{~mm}$ shorter than idea and showing 4-6 mm gingiva (altered passive eruption with suspected slight vertical maxillary excess).

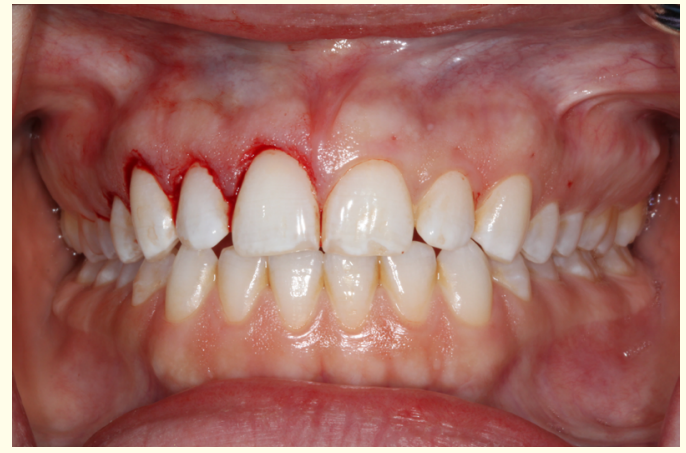

Figure 10 (1)

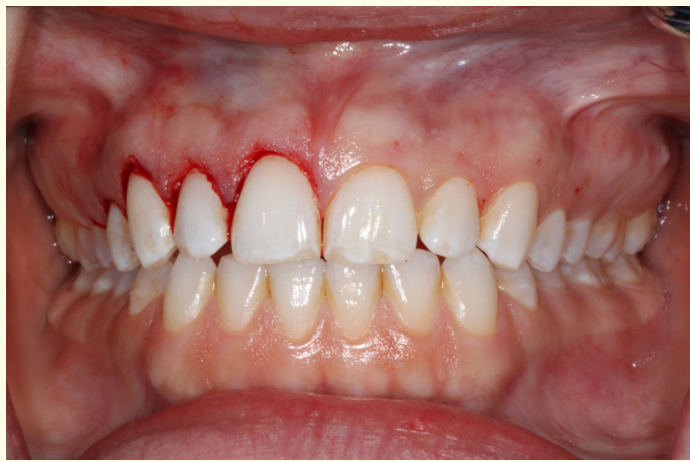

Figure 10 (2)

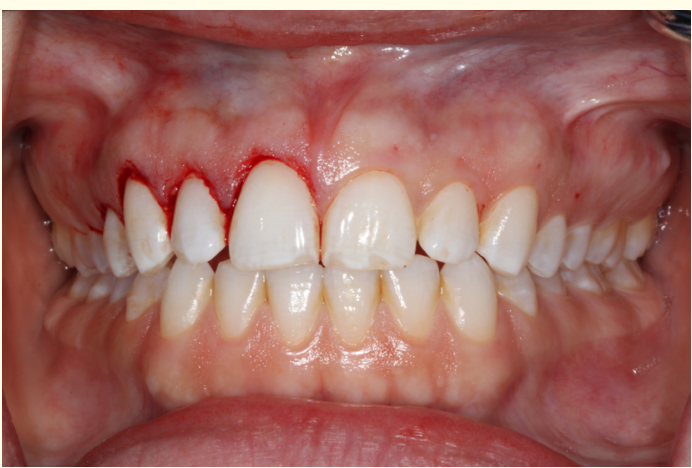

Figure 10 (3)

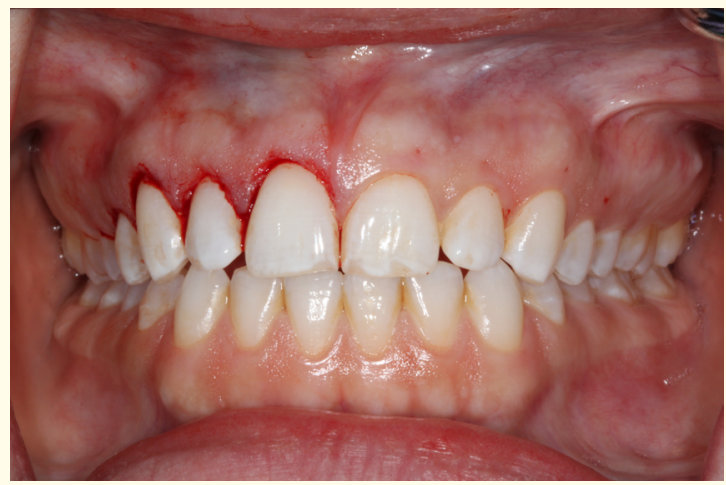

Figure 10: Gingivectomy preformed to establish approximate location of new gingival margin and ideal tooth display. 


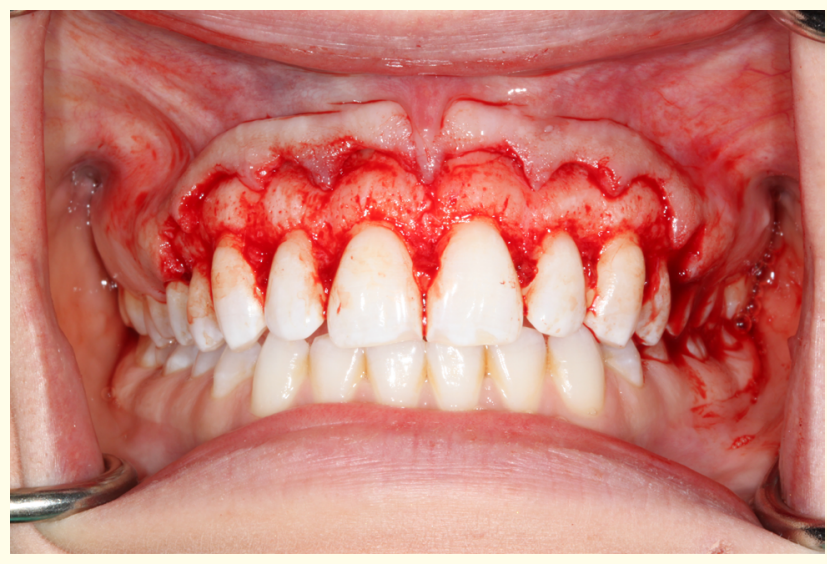

Figure 11: Pre-osseous re contouring with thick maxillary alveolar bone approximating CEJ.

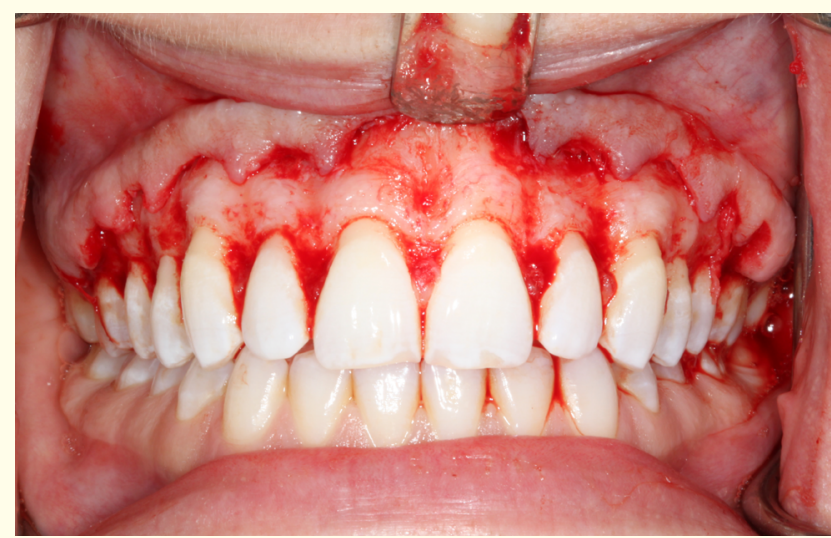

Figure 12: Post-ostectomy and osteoplasty to thin maxillary alveolar bone and position alveolar crest 2.5-3 mm from CEJ.

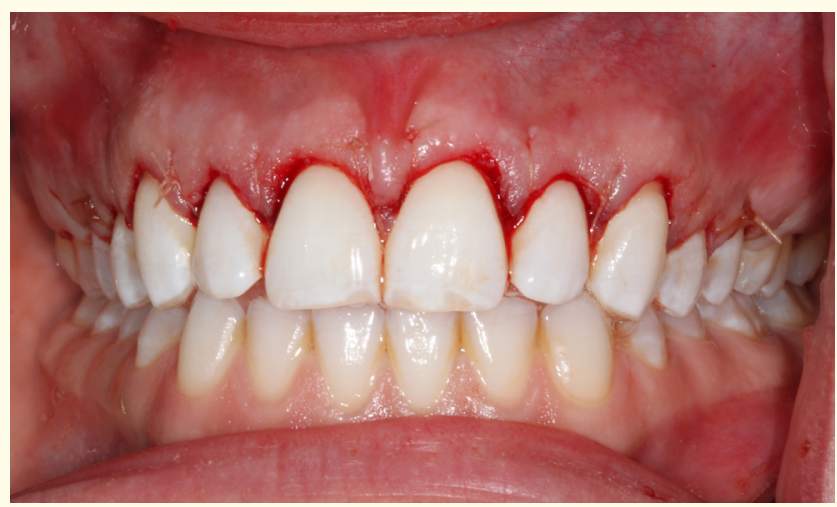

Figure 13: Suturing (single interrupted sling sutures to position margins and interproximal papilla).

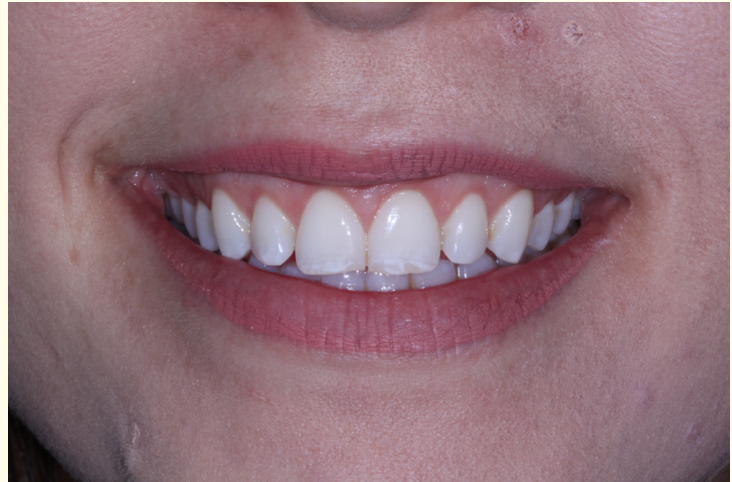

Figure 14: 6 mo. Post-op smile.

Case 3:

26 year old female with chief complaint of "gummy smile". Patient presents excessive gingival display, less than ideal tooth lengths, slight incisal wear, CEJ not detected within gingival sulcus, slightly elongated lower facial $3^{\text {rd }}$ (suspected Vertical maxillary excess, and uneven gingival margins/marginal contours. After clinical examination and facial analysis patient diagnosed with APE and slight VME. Patient was offered esthetic crown lengthening, followed by consultation with OMFS for maxillary impaction (if patient not satisfied with esthetic crown lengthening results). After healing, patient is very pleased with results of crown lengthening procedure and was not interested in additional procedures.

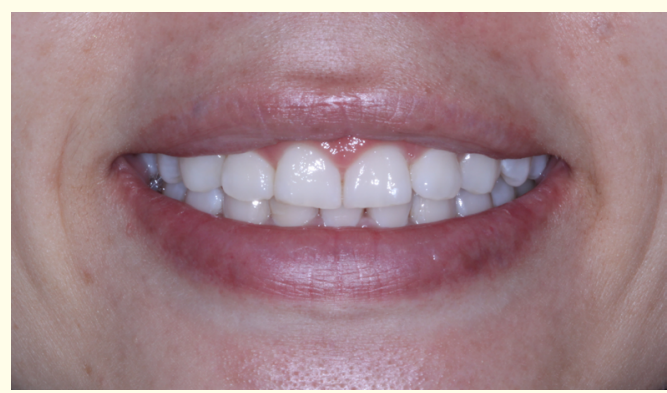

Figure 15: Pre-op smile.

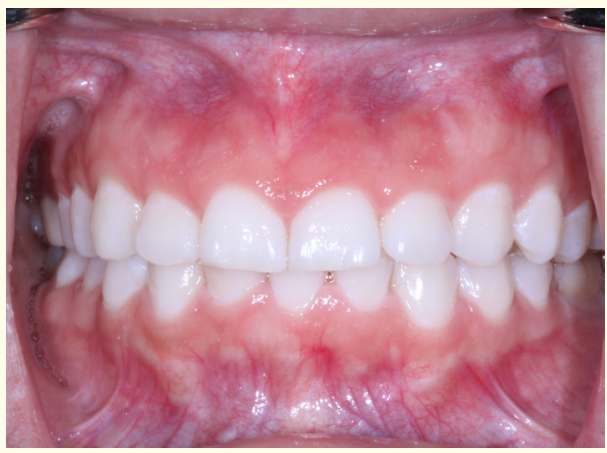

Figure 16: Pre-op retracted. 


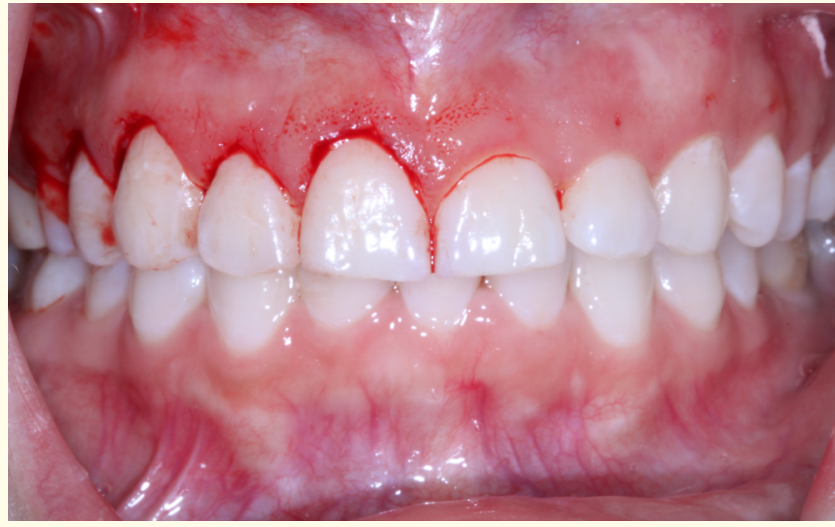

Figure 17: Gingivectomy to establish approximate location of new gingival margins and more idea tooth display.

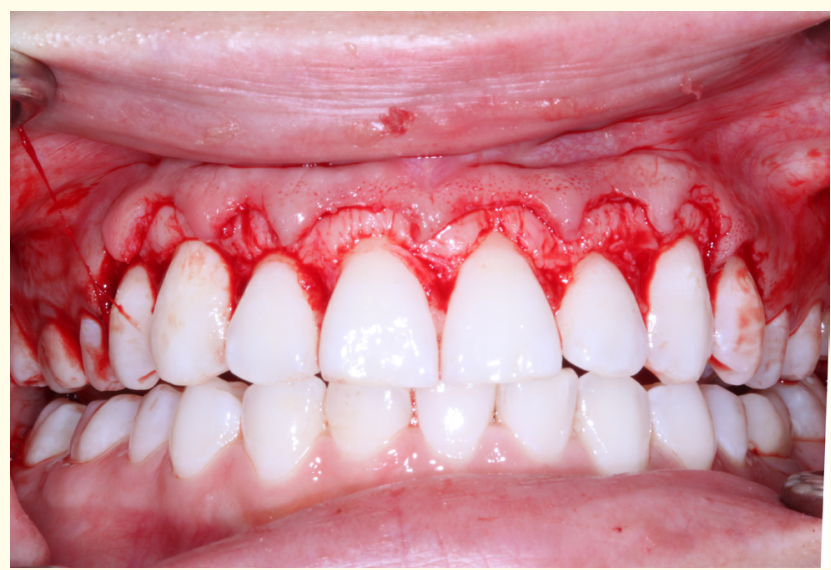

Figure 18: Pre-osseous recontouring.

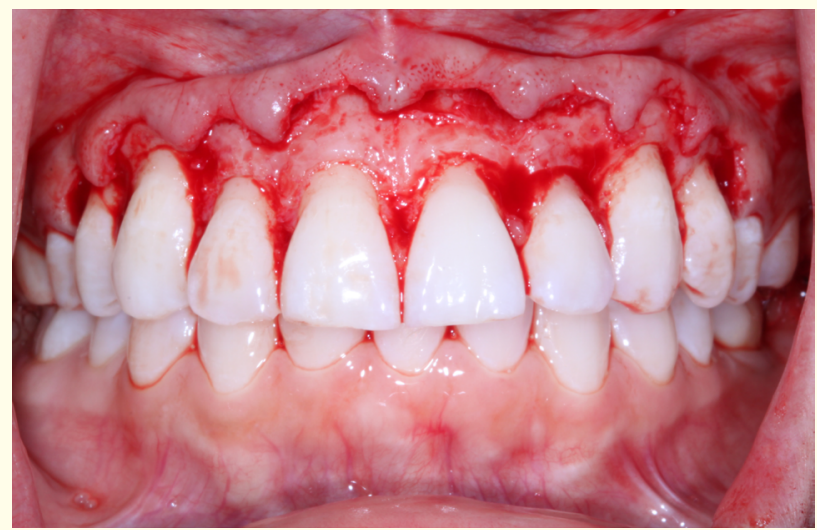

Figure 19: Post-ostectomy and osteoplasty with alveolar crest 2.5-3 mm from CEJ.

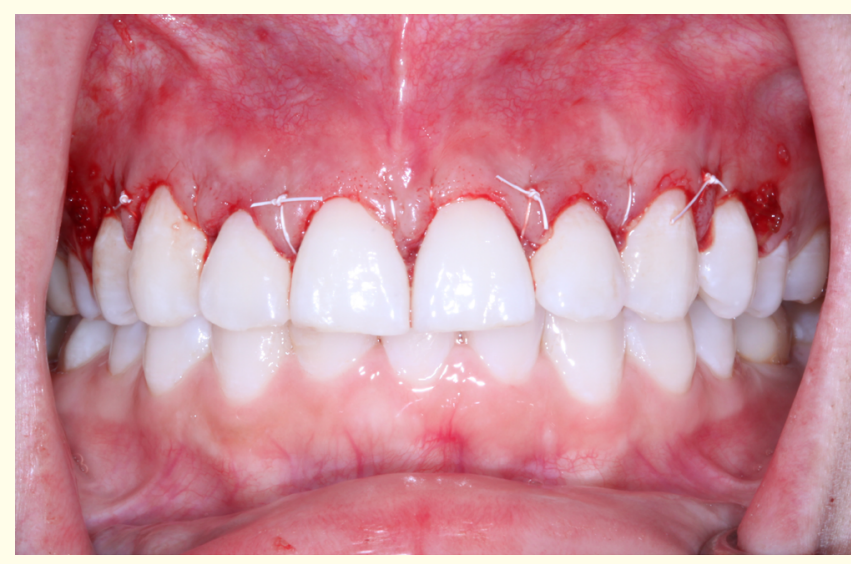

Figure 20: Suturing (single interrupted sling sutures to position margins and interproximal papilla).

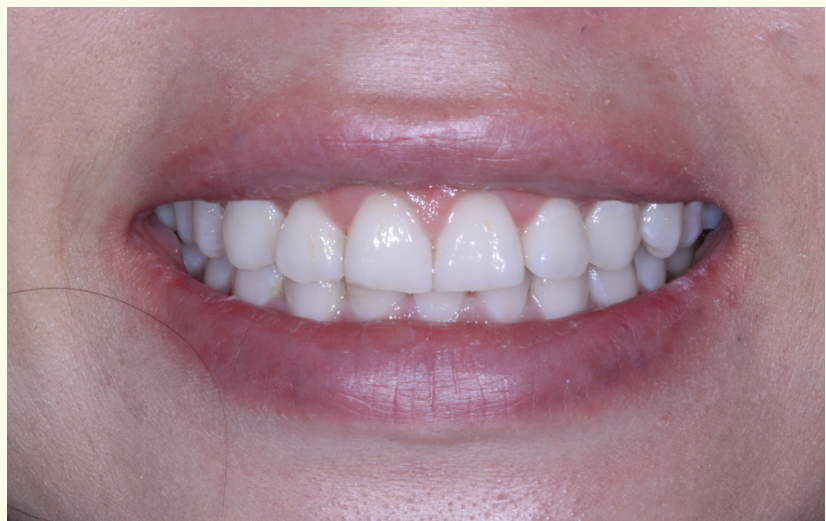

Figure 21: 6 mo. Post-op smile.

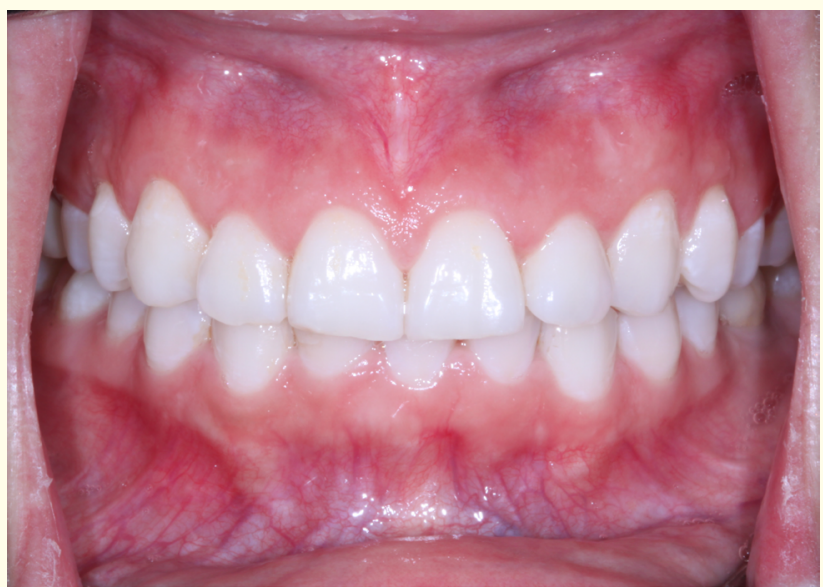

Figure 22: 6 mo. Post-op retracted. 


\section{Conclusion}

A number of treatment options are available for correcting excessive gingival display. However, it is of utmost importance to determine the specific cause for the excess display as it will alter significantly alter the treatment employed. Many a times a combination of gingivectomy, gingivoplasty and osseous resection and/ or lip re-positioning are required. In severe cases, orthognathic surgery may be the only valid option. However, esthetic crown lengthening should always be included in the initial treatment plan to assess the need more complex surgical interventions. Addressing and treating the specific etiological factor will give a satisfactory aesthetic outcome.

However, regardless of the factors contributing to a patient's excessive gingival display, previous authors have recommended $1^{\text {st }}$ achieving ideal display of tooth dimensions. The clinical crown length is dependent on the relation between the incisal edges/cusp tips and gingival margin and can be modified with restorations or surgical crown lengthening. Esthetic crown lengthening procedures effectively expose tooth structure, reduce gingival display, enhance gingival symmetry and gingival contours, reduce thick maxillary (alveolar) bone and lip tension, and most importantly increase self-confidence. Esthetic crown lengthening procedures are less invasive than orthognathic surgical procedures and more proven/predictable than lip repositioning procedures [17] or Botox techniques. If additional procedures are warranted (i.e. impaction), the newly established (proper) tooth dimensions can be used to perfect the patient's smile.

\section{Bibliography}

1. Garber DA and Salama MA. "The aesthetic smile: diagnosis and treatment". Periodontology 200011 (1996):18-28.

2. Levine RA and McGuire M. "The diagnosis and treatment of the gummy smile". Compendium of Continuing Education in Dentistry 18.8 (1997): 757-762.

3. Abhyankar V BP and Hansen J. "Successful management of extensive root caries with gingival recession using a multidisciplinary team approach - A case report of a 1-year follow-up". International Journal of Medical and Dental Case Reports 5.1 (2018): 1-4.

4. Kokich VO Jr., et al. "Comparing the perception of dentists and lay people to altered dental esthetics". Journal of Esthetic Dentistry 11.6 (1999): 311-324.
5. Ker AJ., et al. "Esthetics and smile characteristics from the layperson's perspective: a computer-based survey study". Journal of the American Dental Association 139.10 (2008): 1318-1327.

6. Chu SJ., et al. "Gingival zenith positions and levels of the maxillary anterior dentition". Journal of esthetic and restorative dentistry : official publication of the American Academy of Esthetic Dentistry". Europe PMC 21.2 (2009): 113-120.

7. Armitage GC. "Development of a classification system for periodontal diseases and conditions". Annals of Periodontology 4.1 (1999): 1-6.

8. Tjan AH., et al. "Some esthetic factors in a smile". The Journal of Prosthetic Dentistry 51.1 (1984): 24-28.

9. Vig RG and Brundo GC. "The kinetics of anterior tooth display". The Journal of Prosthetic Dentistry 39.5 (1978): 502-504.

10. Allen EP. "Use of mucogingival surgical procedures to enhance esthetics". Dental clinics of North America 32.2 (1988): 307330.

11. Informational Paper "Drug-Associated Gingival Enlargement". Journal of Periodontology 75.10 (2004): 1424-1431.

12. Peck S., et al. "The gingival smile line". The Angle Orthodontist 62.2 (1992): 91-100.

13. Goldman HM CW. "Periodontal Therapy". Mosby 6th edition (1980).

14. Coslet JG., et al. "Diagnosis and classification of delayed passive eruption of the dentogingival junction in the adult". The Alpha Omegan 70.3 (1977): 24-28.

15. Gargiulo AW., et al. "Dimensions and Relations of the Dentogingival Junction in Humans". Journal of Periodontology 32.3 (1961): 261-267.

16. Nugala B., et al. "Biologic width and its importance in periodontal and restorative dentistry". Journal of Conservative Dentistry 15.1 (2012): 12-17.

17. S PMF Ricardo P., et al. "Does Lip-Repositioning Surgery Improve Long-Term Smile Outcome and Dental Esthetics in $\mathrm{Pa}$ tients with Excessive Gingival Display? A Review of the Current Literature". Clinical Advances in Periodontics 4.4 (2014): 280-287.

\section{Volume 3 Issue 9 September 2019} (C) All rights are reserved by Vrushali Abhyankar., et al. 